Gynäkol Geburtsh Rundsch 1991;32(Suppl 1):64-65

\title{
Mütterliche Todesfälle bei Kaiserschnitt
}

\begin{tabular}{|l|l|l|}
\hline A. & & Beck $^{\mathrm{a}}$ \\
\hline Ch. & & Vutuc $^{\mathrm{b}}$ \\
\hline H.P. & & Friedl $^{\mathrm{c}}$ \\
\hline
\end{tabular}

${ }^{a}$ Geburtsh.-gynäkol. Abt. des Hanusch-Krankenhauses (Vorst.: Prof. Dr. A. Beck)

${ }^{\mathrm{b}}$ Institut für Sozialmed. der Univers. Wien (Vorst.: Prof. Dr. M. Kunze) ${ }^{\mathrm{C}}$ Österreichisches Statistisches Zentralamt

Prof. Dr. A. Beck, Geburtsh.-gynäkol. Abt. des Hanusch-Krankenhauses, Heinrich Collin-Str. 30, A-1140 Wien

Jeder erfahrene Geburtshelfer weiß, daß ein Kaiser-schnitt manchmal zu einer der schwierigsten Operationen unseres Fachgebietes werden kann. In glücklicherweise seltenen Fallen kann es zu tödlichen Komplikationen kommen.

Die Müttersterblichkeit in Österreich hat eine sinkende Tendenz, sie lag 1975 bei 30,9/100.000

Lebendgeborenen und 1990 bei 11,0/100.000 Lg [1].

Für Bayern haben Welsch und Krone [2] eine Müttersterblichkeit von 13,8/100.000 Geburten in den Jahren 1983 bis 1989 angegeben.

Von den 123 Müttersterbefällen in Österreich in den Jahren 1981 bis 1990 traten 42, d. s. 34,1 \% , im Zusammen-hang mit einem Kaiserschnitt auf (Tab. 1). Das bedeutet aber nicht eine kausalen Zusammenhang zwischen Kaiserschnitt und mütterlicher Komplikation, sondern nur, daß die Geburt mit nachfolgendem Tod der Mutter (aus unter-schiedlicher Ursache) durch einen Kaiserschnitt (mit un-terschiedlicher Indikation) beendet worden ist.

Tabelle 2 zeigt durch die Obduktion geklärte, in der kli-nischen Krankengeschichte beschriebene oder in manchen Fallen nur durch die Meldung an das Statistische Zentralamt dokumentierte Todesursachen oder vorausgegangene Komplikationen, soferne sie retrospektiv einigermaßen klärbar waren.

Die Indikation zum Kaiserschnitt bei den Sectiotodes-fallen ist aus Tabelle 3 ersichtlich. Neben fetalen Indikatio-nen (13 Fälle) sind es vor allem die bestehende Grund-krankheit in 9 Fallen, Blutungen in 6 Fallen, EPH-Gesto-se in 4 Fallen, alle anderen Indikationen rücken zahlen-mäßig in den Hintergrund.

Von den 42 Fallen können nur 8 direkt der Operation angelastet werden. Es bestand in diesen 8 Fallen keine be-lastende Anamnese, keine Vorerkrankung oder eine

Schwangerschaftskomplikation: 3 Todesfälle waren durch postoperative Uterusatonie verursacht, in einem Fall eine

Tabelle 1. Die Müttersterbefälle und Sectiotodesfälle in den Jahren 1981-1990

Tabelle 2. Welche Todesursachen oder vorausgehende Komplikationen werden am häufigsten bei Sectiotodesfällen genannt? $(n=42)$ 
Tabelle 3. Die Indikation zum Kaiserschnitt bei den Sectiotodesfällen keine Angaben Placenta praevia vorzeitige Plazentalösung EPH-Gestose, Eklampsie CTGBefund drohende i. u. Asphyxie protrahierte Geburt Amnioninfektionssyndrom bestehende Grundkrankheit Schädel-Becken-Mißverhältnis Beckenanomalie, St. p. Sectio Lagranomalie dystrophes Kind Cyanose der Mutter perforierte Appendix Unfall (Querschnitt

42

Total

postoperative Gerinnungsstörung als Ursache, und in 1 Fall kam es intraoperativ zu einer starken Blutung aus dem Uterus, die nicht beherrschbar war. 2 Fälle traten im Gefolge von Ileus und Peritonitis auf, in 1 Fall kam es zu einer foudroyant verlaufenden Nekrose des Beckenbindegewebes im Wochenbett. Die Indikationenliste in Tab. 3 ist zwangsläufig identisch mit den Indikationen zur Notsectio, die in 0,38 \% aller Geburten zur Anwen-dung gelangt; besonders oft wegen CTG-Pathologien, vor-zeitiger Plazentalösung, Nabelschnurvorfall und Placenta-präviaBlutung [3]. Dabei traten in 18,9 \%, als rund /5 der Fälle intraoperative Komplikationen auf, fast alle durch starke Blutung bedingt. Es ist hier ein Kollektiv unter-sucht worden, welches man den Beinahe-Todesfällen bei der Sectio zuordnen kann.

International üblich wird die Zahl der Todesfälle auf 1.000 Sectiones bezogen (fatality rate). Sie ist natürlich ab-hängig von der Gesamtzahl der Kaiserschnittsoperationen in einem Land und der Relation der elektiven Sectio zur Notsectio. Je höher die Sectiofrequenz ist, umso größer wird der Anteil der elektiven Kaiserschnittsoperationen sein und umso niedriger die fatality rate. Schweden hatte schon 1980 eine fatality rate von 0,4 (Sectiorate 11\%), England/Wales 19791981 ebenfalls 0,4 (Sectiorate 10\%), Bayern 1983-1989 0,49 bei einer Sectiorate von 14,8\%. Für Osterreich läßt sich für das Jahr 1990 bei 90.454 Geburten, einer realistisch geschätzten Sektiorate von 10\% (siehe Daten der Posterpräsentation und ein-zelner Vorträge dieser Tagung) und 3 Sectiotodesfällen eine fatality-rate von etwa 0,3 errechnen.

\section{Literatur}

Beck A, Vutuc Ch, Friedl HP: Müttersterbe-fälle in Osterreich 1975-1989. Gynäkol Rundsch 1991; 31 (Suppl): 166. Welsch H, Krone HA: Über Möglichkeiten und Wege zur weiteren Reduzierung der schwan-gerschaftsbedingten Müttersterblichkeit, Ta-gungsbericht; Geburtsh Frauenheilk 51 (1991)

Beck CT, Klingemann H, Dallacker W, Drä-ger B: Der notfallmäßige Kaiserschnitt -Analyse von 143 Notsectiones. Geburtsh Frauenheilk 52 (1992) 96. 